\title{
Valuing the person's story: Use of life story books in a continuing care setting
}

\author{
Teresa Wills \\ Mary Rose Day \\ Catherine McAuley School of Nursing \\ and Midwifery, University College \\ Cork, Ireland
}

\begin{abstract}
There is an increasing focus on promoting person-centred systems across continuing care settings, emphasizing the need to enhance the quality of life of older adults. Life story books (LSB) can provide a holistic view of older adults, promote relationship-centred care and enhance person-centred care. The process of developing LSB involve collecting and recording aspects of a person's life both past and present. The purpose of this study was to engage residents in developing life story books in a nursing home setting and then to explore the narratives and documented life story books with residents and their families. A qualitative descriptive exploratory design was utilized for the study. Five residents and three family carers participated. Focus groups were tape recorded and thematically analyzed and a review of the LSB was conducted. The central themes from the data analysis related to the social construction of people's lives, social roles and religious values, relationships and loss, and sense of self.
\end{abstract}

Keywords: life story books, older adults, long-term care setting, person-centred care, and relationship-centred care

\section{Introduction}

Like many other countries in the World, Ireland has a rising older population and this demographic shift has meant that older people are seeking placement in a variety of continuing care settings, such as nursing homes or community hospitals. Older people tend to be stereotyped as a uniform group with total disregard for individuality (National Council for Ageing and Older People 2005). Much has been written about the importance of person-centered care, older people and quality of life (Packer 2000; McCormack 2004; Nolan et al 2004). A person-centered approach to care requires practitioners to learn more about an individual's life story, in order to seek meaning and understanding of the older person's life experiences and attitude (William and Grant 1998; McCormack 2004). Life story books (LSB) can give people a sense of identity and provide a more holistic view of older adults, and offers the potential to enhance person-centered care (Clarke et al 2003).

\section{Life story books}

McKeown and colleagues (2006 p. 238) defined life story work (LSW) as "a term given to biographical approaches in health and social care settings that gives people time to share their memories and talk about their life experiences". LSB, also called life books, can consist of photographs, written accounts in people's own words, materials relating to a person's life and life history, resulting in the collection of information to form the LSB (Heathcode 2005). The photographs and memorabilia can be used therapeutically to enhance communication between health professionals, residents, caregivers and their families. These LSB can highlight a resident's unique life history (Hewitt 2000), and be used as a basis for individualized care (Hansebo and Kihlgren 2000). Anderson and colleagues (1992) suggested that it can help to improve assessment and 
assist in the building of relationships as people listen to each other's stories (Hansebo and Kihlgren 2000). LSW has the potential to provide a greater insight into a person's needs and behaviors (Johnson 1976; Clarke et al 2003), providing people with a voice and it may help challenge attitudes and beliefs about people (Meninger 2006).

Hirst and Raffin (2001) described stories as threads that weave together the life of an older adult, and are the raw material from which the sense of self emerges. Autographical storytelling in the form of a LSB can give shape to life experiences and may help to understand memories in new ways, as the story is both confirmed and recreated (Lea Gaydos 2005). The creation of a life story book is an important component in a person's identity and in the promotion and maintenance of self(Surr 2006). Transition to care such as a nursing home can present many challenges for older adults, as it can create a sharp break with their previous life, resulting in feelings of isolation and anonymity, loss of identity and loneliness (Heliker and Jaquish 2006). Heliker (2007) supports the view that a culture of listening and story sharing can promote a caring environment for residents in a continuing care setting. LSB can promote and enhancing person-centered care and relationship-centered care (Nolan et al 2004).

The purpose of this study was to introduce LSB into a nursing home setting as part of a quality improvement project.

\section{Theoretical framework}

Narrative theories provided the conceptual framework for this study. Narrative approaches can include autobiography, biographies oral history and autoethnography (Holloway and Freshwater 2007). Narration is part of everyday life and people have a desire to tell their stories and it is the stories people tell themselves and others, about their past and present lives, which helps to make sense of their life experiences. A person's identity is what provides the person with a sense of continuity, a sense of coherence and a sense of self. Drawing together the different life experiences helps to shape and give meaning to life as a whole, and this is called the Theory of Narrative Identity (Polkinghorne 1996; Roesler 2006). People are active in the construction of their own story. Lives are shaped by social circumstances, political opinion, religion, economic and ideological climate (Holloway and Freshwater 2007). People's experiences are shaped by the relationships they have with family friends, social groups communities, and this in turn is influenced by culture. Járvinen (2004) maintains that the focus of the narratives is important, as individuals centre their stories around significant experiences.
Descriptive narratives and photographs in the form of LSB offer opportunities to record events and accounts of a person's life experiences, and reveal a snapshot of the person and their individual story.

\section{Methodology}

\section{Aim}

The aim of the study was to engage residents in developing LSB in a nursing home setting, and then to explore the narratives and documented LSB with residents and their families.

\section{Life story book project}

The Director of Nursing and staff approached 10 residents and invited them to attend an information evening in the nursing home with family, staff and researchers. Information on the study and schedule plan was given by the research team to residents' families and staff. A completed life story book was available for viewing. Six residents with their families agreed to participate in the project. An information pack with supporting material to compile the life story book was given to each resident. This included a ring bound photograph album to allow for the insertion of photographs, CD's, stories, newspaper cuttings and memorabilia. A statement of privacy and confidentiality was placed in the first page of each book. Prompts were given as guidelines on headings for inclusion in the LSB, but residents were free to decide how they wished to tell their story. Support was provided to residents and families to scan, laminate photographs and memorabilia, and to type up stories by nursing home staff over a three month period.

\section{Ethical approval}

Ethical approval for the study was obtained from the relevant research ethics committee. A detailed consent process was in place to ensure residents and their families were fully informed of the study. It was agreed that ownership of the life story book was with the residents and their families

\section{Data collection and analysis}

A qualitative descriptive design was used (Morse and Field 1996). Six residents ( 3 male, 3 female) aged 80 to 91 years, resident in the nursing home setting from 6-24 months, participated in focus groups in September 2006. Interviews were tape recorded with the participant's permission. Each resident was asked about their life story book, and all participants were asked about engaging in life story book project. 
Residents $(n=6)$ eagerly narrated and shared specific memories and experiences that they had documented in the life story book. During the interview, field notes were made by one of the researchers using a flip chart. In addition, permission was given to take away the LSB for review by the research team.

The information from both the interview transcripts, field notes and LSB were read and re read, and detailed notes were made. A documentary review of the narratives, photographs and memorabilia included in the LSB provided insight into the different life experiences of each resident. They were then subjected to thematic analysis using Burnard's (1991) nine stage processes to analyze data, identify patterns and unique themes, commonalities, and differences in the participant's responses. Themes were identified by 2 researchers independently, and then merged to form a joint understanding of the data. A full audit trail of the data has been retained for inspection.

\section{Findings}

Four central themes emerged from the analysis of data from interview transcripts and review of the LSB . These related to the social construction of people's lives, social roles and religious influence, relationships and loss, and sense of self.

\section{Theme I: Social construction of people's lives}

All participants preferred to think about the past, rather than the present, and each identified the past as being a very different time. Each of the LSB captured rich accounts of life growing up in Ireland at the turn of the century. All residents recalled their critical life memories in detail - places of birth, parents, siblings, relationships, family life, memories of school, and growing up. These experiences were explored in terms of the cultural context and times in which they lived. This was portrayed using historical photographs, family photographs and memorabilia, together with descriptive narratives and stories. There were no modern conveniences, no electricity, television, or toilets, and transport was by a horse and cart, bicycle or on foot. Daily life was described by one resident:

"Went to school barefoot and ran home to do our jobs...helped in the house and on the farm... cycled to the local town to get the flour to bake the bread... milked the cows and made the butter. We learned to knit, sew and mend."

A number of participants referred to the common use of the Irish language and shean fhocials, and the need to pass these on to their families as part of their heritage.
Work was scarce and emigration had touched the lives of participants and their families. with people emigrating to the United Kingdom and America and not returning for many years. Each of the residents had been born into a large family, and each spoke about being part of a large extended family. Grandparents and extended families lived in the same houses, and women stayed at home rearing children and keeping house, creating close ties among family members. Tuberculosis and polio were common illnesses at this time, and had affected the lives of two of the participants.

Life was simple but economic problems of the time, such as low wages and dependence on one income, meant there was little money. This was described by one resident:

"Luxuries were few but we also had the essentials. There was less heavy drinkers and little or no violence."

Participants reminisced about dancing at the crossroads, house stations, thrashings, listening to live music and storytelling. Overall, they felt life had been simpler in those days and portrayed fond memories of those times. Residents constructed their lives as being hard and simple, yet at the same time there were no expectations, and people were happy and content.

\section{Theme 2: Social role and religious influence}

All six participants discussed social roles related to work and recounted details of their past occupations, and the contribution they made to society and family during their life. Participants left school at an early age, went working, and recalled their life stories in the context of the cultural values associated with living in rural Ireland. All participants acknowledged that times were hard, but circumstances were similar for everybody and everybody helped each other out. One resident recalls:

"Times were hard for people, many left at twelve years of age for economic reasons but, somehow, we always managed to get by, without owing anybody anything."

The majority of people at that time were living on small farm holdings, and women were usually responsible for the care of livestock, poultry and pigs. Roles or jobs described including churning the butter, rounding up the cows and calves, bringing the water from the well, bringing the turf for the fire, feeding the pigs or hens, saving the hay, selling the eggs, buying the day old chicks that came in the box on the bus, and then rearing them. One resident recollected:

"I cycled to the bog 4 miles away to save the turf, our fuel for the winter months. Lots of people worked in the bog and we had great conversations." 
Some of the favorite pastimes described included the following: soccer, bowls, reading, writing, storytelling, acting, sing songs, chats, listening to radio shows, music, golf and gardening.

Religion appeared to have been an important and imposing influence in shaping people's social lives, and was a recurrent theme in the narratives and the LSB. The following quotation makes this very evident:

"The church had an imposing influence on people's lives during the 1940s, 1950s and 1960s and 1970s. The church dominated our lives."

The importance of religious practices and symbols were mentioned by five of the residents and included the rosary, mass, confession, Stations of the Cross, holy pictures, confirmation, communion and baptism. One resident referred to "being deeply religious with a very keen sense of right and wrong".

Another resident spoke about going to daily mass with her husband, and the central part that faith played in their lives, as well as "the need to pass this on to their children". The LSB provided a snapshot view of the cultural and religious beliefs and practices of each individual resident. Religion still plays a very important and central part in residents' lives. The social role that religion plays is epitomized in the following statement:

"My faith is as strong as ever. I rarely miss Mass and say the rosary every night with a few friends in my new home."

LSB could be used to support assessment and provide information to support the expressed needs and wishes of residents.

\section{Theme 3: Relationships and loss}

Relationships with others, and particularly with family members, played a significant role in the lives of each of the residents. Relationships and ties with family members were the most significant. Each spoke about their parents and their siblings, and one of the participants spoke about the big influence his grandfather had on his life.

"I adored Granda, an old seadog, and although he died when I was eight, he was and is my role model. I think it was his independence of spirit."

The majority of residents married and had children, grandchildren, great grandchildren and extended families. All discussed their relationships with them during the narrative, and included pictures of them in the LSB. Five of the six residents spoke about the new relationships and friends they had made since coming to their new home (nursing home).
One of the health professionals commented that "looking at the book allowed me to share my memories with residents."

Loss and death are part of everyone's life experiences. A number of the residents touched on this in the LSB and narrative. Several spoke in particular about the loss of their husbands and wife, and the huge support that had been given by their families during that time and now. This is communicated in the following comment:

"I was lost without my life's partner. He was a wonderful

husband and father whom I loved dearly. I was very proud of him and this life story book is a tribute to him."

One resident had included a photograph of his daughter, who was deceased, but he did not speak about this in the narrative or life story book. We can only assume that it was difficult for him to talk about this. One resident had difficulties in relation to property, which precipitated transition to care, but time has helped to heal loss and family disagreements. Photographs and memorabilia from his home played a very important part in his life story.

The narratives and LSB allowed those present to see each person through their story as a member of a community, as a grandfather, father, mother, sister and friend.

\section{Theme 4: Sense of self}

The LSB provided the residents with the opportunity to share stories, pictures and memorabilia's with other participating resident's family and staff. The key themes that transcended all the stories provided a coherent connection between selected life events, self, other residents and staff. The creation of the book helped residents reflect on past life experiences, family, relationships, home and allowed them to validate their self-identity. Narrating and documenting their life story was a therapeutic process, and gave them the opportunity to affirm themselves and their sense of self. This was captured in the following comment:

"I feel that I have generally lived a rich life alternating between quiet desperation and high level frolicking. As I look back on my life I feel much fulfilled."

Personal experiences and achievements, as well as dreams and hopes for the future, were included by some of the residents in the life story book. One resident typifies this, "looking forward to seeing a bit more of life and seeing my grandchildren get married", while another spoke about "wanting to see more of life yet".

The coming alive of old memories, stories and emotions was extremely satisfying for residents, families and carers. The stories told challenge ageist attitudes, and show that 
older people have much to contribute to society. Residents also realized they had interesting stories to share with other residents, family and staff that could be used to enhance social interaction.

\section{Discussion and conclusion}

The research findings from this study have important implications for the care of older adults. The narratives and stories portrayed the cultural values, social roles and relationships of each individual resident. The LSB brought people back in time, and captured the world residents grew up in 80-90 years ago. People's past was identified as a very different time and several of the residents captured and portrayed this using photographs and memorabilia. All participants narrated and spoke about their earlier years, school days, occupation, family, friends, and relationships, and the contributions they made to family and society. These critical life memories and stories provided insights into the uniqueness of each resident's identity, and captured details of their life experiences. LSW has the potential to provide a greater insight into people's needs and behaviours (Clarke et al 2003; Johnson 1976) and has the potential to make strong connections with the person's past and present lives.

All participants discussed social roles related to work, and recounted details of their past occupation. This gave participants a sense of importance about their contribution to society. Several of the participants left school at an early age, and recalled their life stories in the context of the cultural values associated with living in rural Ireland and the difficulties they encountered. In clinical practice, health care professionals tend to focus on physical needs when people are admitted to continuing care settings, and files and nursing care plans do not capture characteristics of resident's values and culture (Coker 1998). Knowledge of a resident's life history can improve nursing care and attitudes of people (Reichmann et al 2004).

Spirituality and religion played an important part in resident's past and present lives, and had an important influence in shaping people's values and roles. A holistic view of each resident was captured within the stories, allowing those present to see the person in the context of his or her past, rather than the context of the care setting. Clarke and colleagues (2003) advocated that life stories can help health care professionals to see the person behind the illness, and have the potential to promote person-centred care. This is supported by Kenyon (2003), who states that residents should not be seen in the context of their disease, but viewed as a unique person with a life story.
Relationships with other people and family played a significant part in the narratives and the LSB. A number of participants spoke about the death of their parents, husband or wife, and the impact and change this loss had on their lives. People's experiences were shaped by their relationships with family, friends, social groups and communities, both in the past and the present. Some residents articulated how happy they were in their new home, and with the new relationships and friends they had made. LSB and storytelling are one way of promoting relationships between families, residents and staff, and may help to create a caring environment (Heliker 2007). Engaging residents in the development of LSB may provide opportunities to promote and enhance person-centered care and relationship-centered care (Nolan et al 2004).

There was a deep and rich context to the stories, and commonalities across cultural values and past allowed those present to connect with each other. Residents drew on memories to construct stories about themselves, which allowed them to validate their self-identity. Engaging older people and families in LSW is the most meaningful way of preserving family history and historical memories for future generations (Elford et al 2005). Reviewing the photographs, documents and meaningful objects captured the context of the older person's life, and represented aspects of their self identity. The inclusion of important certificates and memorabilia by residents gave them a forum to portray these symbols of self. Hirst and Raffin (2001) described stories as threads that weave together the life of an older adult, and are the raw material from which the sense of self emerges. Hewitt (2000) has advocated the construction and reconstruction of a life story as a critical part in the development of self and may assist in transition to continuing care settings.

The findings from this study have important implications and provide insights into the importance of life storybooks in supporting the delivery of quality person-centred care. In the field of gerontological nursing, adopting a person-centred approach to practice requires us to work with older people's beliefs, values, wants, needs and desires. The challenge for health care professionals is to get to know the individual as a person, uncover what makes that person unique and to individualize the care provided.

There is no set way to developing LSB. Older people are not a homogenous group with depleted writing and storytelling skills, and this has been challenged in this study. LSB can be developed by a person themselves, family carer or health professional. Creating the LSB was a challenge, and required the support and involvement of families, staff and researchers. 
The researcher's role in the project was to facilitate the process and promote the value of LSB to staff family and residents. Family carers and care assistants supported and involved the resident in the creation and development of the life story book. Policies and guidelines for the development of LSB need to be developed with management and staff before introducing LSB in practice (Day and Wills 2007).

It is acknowledged that this was a small study and limits the generalization of findings. However, this study suggests a number of directions for future work in this area. Future research is needed to expand the findings from the present study, and to include people with dementia, older people with learning disabilities and people suffering from chronic disease. In particular, future research should include health care professionals and elicit their views and experiences on the use of LSB in clinical practice.

The use of LSB in this study gave a voice to older people, and gave them the opportunity to write and narrate their story. It allowed them to share their stories with residents, family and staff. LSB make an important contribution in meeting the individual needs of older people. Within the continuing care setting, coming to know and connecting with older people using LSB needs to be encouraged. It is important that these care facilities support LSB, and engage staff and residents in its use.

\section{Acknowledgments}

The authors would like to acknowledge the contribution of the participants in this study. Since the completion of the pilot study one of the participants has died. The author wish to acknowledge Ms Geraldine Keohare, Director of Nursing in nursing home. There are no conflicts of interest to report.

\section{References}

Anderson KH, Hobson A, Steiner P et al. 1992. Patients with dementia: Involving families to maximise nursing care. J Gerontol Nurs, 18:19-25.

Burnard P. 1991. A method of analysing interview transcripts in qualitative research. Nurse Educ Today, 11:461-6.

Coker E. 1998. Does your care plan tell my story? Documenting aspects of personhood in long term care. J Holist Nurs, 16:435-52.

Clarke A, Hanson E, Ross H. 2003. Seeing the person behind the patient: enhancing the care of older people using a biographical approach. J Clin Nurs, 12:697-706.
Day MR, Wills T. 2007. Mind matters. World Ir Nurs, 15:35-6.

Elford H, Wilson F, McKee KJ. 2005. Psychosocial benefits of solitary reminiscence writing: An exploratory study. Aging Ment Health, 9:305-14.

Hansebo G, Kihlgren M. 2000. Patient life stories and current situation as told by carers in nursing home wards. Clin Nurs Res, 9:260-79.

Heathcode J. 2005. Part two: Choosing and individual reminiscence approach. Nurs Residential Care, 7:78-80.

Heliker D. 2007. Story sharing: Restoring the reciprocity of caring in long-term care. J Psychosoc Nurs Ment Health Serv, 45:20-3.

Heliker D, Jaquish A. 2006. Transition of new residents to long-term care: Basing practices on residents perspective. J Gerontol Nurs, $32: 34-42$.

Hewitt H. 2000. A life story approach for people with profound learning disability. Br J Nurs, 9:90-5.

Hirst S, Raffin SR. 2001. Contemplating, caring, coping, conversing: A model for promoting wellness in later life. J Gerontol Nurs, 30:16-21.

Holloway I, Freshwater D. 2007. Narrative research. Nursing Oxford: Blackwell Publishing.

Jarvinen M. 2004. Life histories and the perspective of the present. Narrative Inq, 14:45-68.

Johnson M.L.1976. That was your life: A biographical approach to later life. In: Carver C, Liddiard P. (ed). An ageing population. Sevenoaks: Hodder and Stoughton, pp. 98-113.

Kenyon GM. 2003. Telling and listening to stories: creating a wisdom environment for older people. Generations, 27:31-3.

Lea Gaydos H. 2005. Understanding personal narratives: an approach to practice. $J A d v$ Nurs, 49:254-9.

McCormack B. 2004. Person-centeredness in gerontological nursing: an overview of the literature. Int J Older People, 13:31-8.

McKeown J, Clarke A, Repper J. 2006. Life story work in health and social care: systematic literature review. J Adv Nurs, 55:237-47.

Meininger HP. 2006. Narrating, writing, reading: life story work as an aid to self advocacy. J Learn Disabl, 34:181-8.

Morse JM, Field PA. 1996. Nursing research: The application of qualitative approaches. 2nd ed. London: Chapman and Hall.

National Council on Ageing and Older People. 2005. Perceptions of ageism in health and social services in Ireland. Dublin: National Council on Ageing and Older People.

Nolan MR, Davies S, Brown J, et al. 2004. Beyond ‘person-centered' care: a new vision for gerontological nursing. Int J Older People, 13:45-53.

Packer T. 2000. Series - obstacles to person-centered care delivery. Part 1: Does person-centered care exist? J Dement Care, 8:19-21.

Polkinghorne D. 1996. Explorations of narrative identity. Psychol Inq, 7:363-67.

Reichman S, Leonard C, Mintz T, et al. 2004. Compiling life history resources for older adults in institutions: Development of a guide. $J$ Gerontol Nurs, 30:20-8.

Roesler C. 2006. A narratological methodology for identifying archetypal story patterns in autobiographical narratives. J Anal Psychol, 51:574-86.

Surr C. 2006. Preservation of self in people with dementia living in residential care: A socio-biographical approach. Soc Sci Med, 62:1720-30.

William B, Grant G. 1998. Defining 'people-centeredness': making the implicit explicit. Health Soc Care Community, 6:84-94. 